\title{
Title: Using high-resolution contact networks to evaluate SARS-CoV-2 transmission and control in large-scale multi-day events
}

\author{
Authors: Rachael Pung ${ }^{1-3 *}$, Josh A Firth ${ }^{4,5}$, Lewis G Spurgin ${ }^{6}$, Singapore CruiseSafe working \\ group, CMMID COVID-19 working group, Vernon J Lee ${ }^{1,7}$, Adam J Kucharski ${ }^{2,3}$
}

\author{
Affiliations: \\ ${ }^{1}$ Ministry of Health, Singapore \\ ${ }^{2}$ Centre for the Mathematical Modelling of infectious Diseases, London School of Hygiene and \\ Tropical Medicine, London, UK \\ ${ }^{3}$ Department of Infectious Disease Epidemiology, London School of Hygiene and Tropical \\ Medicine, London, UK \\ ${ }^{4}$ Department of Zoology, University of Oxford, Oxford, UK \\ ${ }^{5}$ Merton College, University of Oxford, Oxford, UK \\ ${ }^{6}$ School of Biological Sciences, University of East Anglia, Norwich, UK \\ ${ }^{7}$ Saw Swee Hock School of Public Health, National University of Singapore, Singapore
}

${ }^{*}$ Corresponding author. Email: rachael.pung@Ishtm.ac.uk

Abstract: The emergence of the highly transmissible SARS-CoV-2 Delta variant has created a need to reassess the risk posed by increasing social contacts as countries resume prepandemic activities, particularly in the context of resuming large-scale events over multiple days. To examine how social contacts formed in different activity settings influences interventions required to control outbreaks, we combined high-resolution data on contacts among passengers and crew on cruise ships with network transmission models. We found passengers had a median of 20 (IQR 10-36) unique close contacts per day, and over $60 \%$ of their contact episodes were made in dining or sports areas where mask wearing is typically limited. In simulated outbreaks, we found that vaccination coverage and rapid antigen tests had a larger effect than mask mandates alone, indicating the importance of combined interventions against Delta to reduce event risk in the vaccine era.

\section{Main Text:}

Many countries are resuming domestic activities as vaccination coverage and population immunity against SARS-CoV-2 increases (1-3). Settings with particularly high contact rates, such as meetings, conferences, exhibitions, and cruises, are also revenue generating sectors with high pre-pandemic visitor throughput across the world $(4,5)$. However, the transmission dynamics on real world networks of large-scale events are yet to be fully explored in the COVID19 era (6). Understanding the risk of outbreaks in these settings and possible outbreak control interventions would enable event planners to gauge the sustainability of their operations and for policy makers to weigh the public health cost against the economic gains. Given breakthrough infections in vaccinated individuals and the spread of the highly transmissible SARS-CoV-2 Delta variant (7-9), countries have employed a range of tools alongside routine vaccination to suppress disease transmission, including vaccine certifications, rapid antigen tests, mask 
mandates, and digital contact tracing devices $(10,11)$. Although there have been efforts to estimate infection risk during large events from routine testing data and contact tracing interviews (12), data from contact tracing devices can enable finer scale assessment of interactions such as the distance and duration of contact depending on the strength and continuity of the Bluetooth signals captured in these devices. Furthermore, these devices overcome the challenges of recall bias and achieve more reliable estimates of the contacts in a network (13).

In Singapore, 'cruises to nowhere' (i.e. cruises that depart and return to the port of origin without other ports of call) began as a safe travelling option during the COVID-19 pandemic with a range of activities and hence setting-specific interactions onboard. We collected contact data from around 1,000 crew and 1,300 passengers per sailing between November 2020 and February 2021 and analysed the resulting social interaction networks. We then use these contact networks to simulate SARS-CoV-2 Delta variant outbreaks and assess how different combinations of interventions and network formulations influence transmission in a range of settings during a large-scale event.

\section{Results}

\section{Characterising social interactions on cruise ships}

3,963,256 contact episodes with 1,846,312 unique contact pairs were recorded during 37-hour data collection periods across four separate three-day sailings (see Materials and methods). During the period studied, cruise lines were operating at 50\% capacity with a passenger to crew ratio of approximately $1: 1$ and passengers from different travelling groups were strongly advised to maintain a physical distance of at least one metre from other groups.

The four sailings had a mean of 1304 passengers (range 1142-1682) with a median age of 54 (IQR 35-63) and a mean of 1050 crew (range 1003-1083) spread across eight work departments (Table 1). There was a high density of contacts among passengers, with some clustering of contacts among crew, although crew members may be required to work with other individuals from the same or different departments, and roles such as housekeeping and galley crew had contacts dispersed across the network (Fig. 1A and fig. S1). Crew were encouraged to form 'work bubbles' as part of COVID-19 workplace interventions (i.e. team of workers that work independently from another team). As a result, on average they had 10 unique close contacts per day (IQR 6-18), about 50\% lower than that of passengers (median 20, IQR 10-36) (Fig. 1B). If the threshold for close contact (defined as a cumulative duration of interaction of 15 minutes in our baseline analysis) was relaxed to a shorter duration, the overall median unique close contacts scaled exponentially (Fig. 1C). The strength of each contact (i.e. edge weights) can be further quantified as a function of the duration of the contact (see Materials and methods). Adjusted for the duration of each contact, the median weighted degree in crew was 8.3 (IQR 4.4-13.5), while the median in passengers was 13.9 (IQR 5.6-23.7) (Table 2). Furthermore, passengers had significantly higher connectivity with other highly-connected individuals, with a median eigenvector centrality of 0.3 (IQR $0.1-0.5$ ) compared to a median of 0.09 (IQR 0.03-0.2) for crew (Table 2 and fig. S2).

Table 1 Demographics of passengers (A) and crew (B) onboard four cruise sailings. Number of passenger and crew presented as mean with range in brackets, unless specified otherwise. 
medRxiv preprint doi: https://doi.org/10.1101/2021.11.12.21266183; this version posted November 14, 2021. The copyright holder for this preprint (which was not certified by peer review) is the author/funder, who has granted medRxiv a license to display the preprint in perpetuity.

It is made available under a CC-BY 4.0 International license.

1

\begin{tabular}{|l|l}
\hline$A$ & $\begin{array}{l}\text { Passenger }=1304 \\
(1142-1682)\end{array}$ \\
\hline
\end{tabular}

\begin{tabular}{l|l}
\hline Demographics & \\
\hline
\end{tabular}

Median age across all

sailing in years (IQR)
$\begin{aligned} & \text { Passengers by age } \\ & \text { group }\end{aligned}$

\begin{tabular}{|l|l|}
\hline$<12$ years & $47(36-61)$ \\
\hline $12-29$ years & $166(123-285)$ \\
\hline
\end{tabular}

\begin{tabular}{|l|l|}
\hline $30-39$ years & $184(99-327)$ \\
\hline $40-49$ years & $164(144-199)$ \\
\hline
\end{tabular}

\begin{tabular}{|l|l|}
\hline $50-59$ years & $285(268-317)$ \\
\hline $60-69$ years & $314(274-336)$ \\
\hline$\geq 70$ years & $146(95-176)$ \\
\hline Gender & \\
\hline Female & $676(602-832)$ \\
\hline Male & $625(540-850)$ \\
\hline
\end{tabular}

2

* Details of each department are provided in supplementary table 1.

\begin{tabular}{|l|l|}
\hline B & $\begin{array}{l}\text { Crew }=1050 \\
(1003-1083)\end{array}$ \\
\hline Department & \\
\hline Entertainment & $77(73-81)$ \\
\hline $\begin{array}{l}\text { Food \& Beverage } \\
(\text { F\&B })\end{array}$ & $179(171-185)$ \\
\hline Galley & $214(208-219)$ \\
\hline Gaming & $175(163-187)$ \\
\hline Hotel services & $84(77-92)$ \\
\hline Housekeeping & $123(114-137)$ \\
\hline Marine & $154(148-160)$ \\
\hline Security & $44(40-48)$ \\
\hline &
\end{tabular}

3

4

5 
medRxiv preprint doi: https://doi.org/10.1101/2021.11.12.21266183; this version posted November 14, 2021. The copyright holder for this preprint (which was not certified by peer review) is the author/funder, who has granted medRxiv a license to display the preprint in perpetuity.

It is made available under a CC-BY 4.0 International license .

A

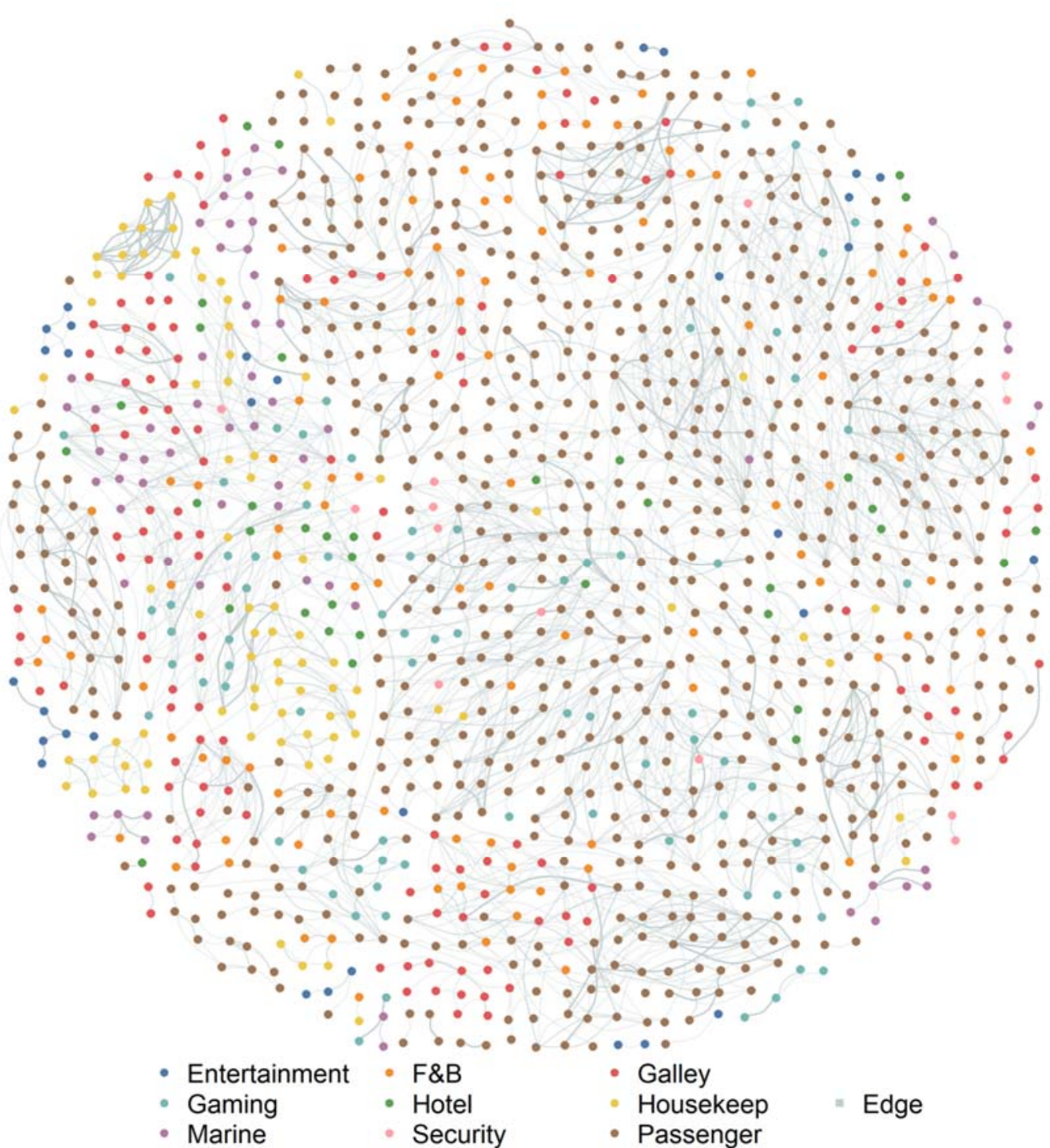

$\mathrm{B}$

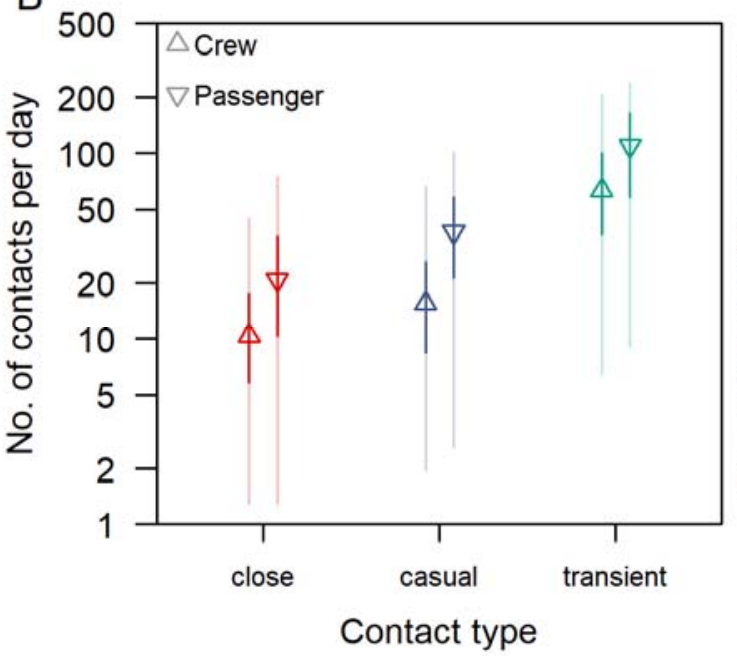




medRxiv preprint doi: https://doi.org/10.1101/2021.11.12.21266183; this version posted November 14, 2021. The copyright holder for this preprint (which was not certified by peer review) is the author/funder, who has granted medRxiv a license to display the preprint in perpetuity.

It is made available under a CC-BY 4.0 International license .

Fig. 1 Distribution of cruise ship contacts. (A) Illustrative short-term network dynamics, showing cumulative network of all contacts that began between 12.00 to $12.05 \mathrm{pm}$ on the second day of a sail and lasted till the end of their contact episode. Edge width and colour intensity are a function of the type of contact (i.e. close, casual and transient). Intra- and inter-cohort contacts are represented by the connection of nodes with the same and different colour respectively. (B) Number of unique close, casual, transient contacts made per day by passenger and crew. (C) Number of close contacts per day for both crew and passengers using different thresholds for the cumulative duration of interaction.

Table 2 Network properties of passengers and crew onboard four cruise sailings. Results presented as median with IQR in brackets.

\begin{tabular}{l|l|l|l|}
\hline Network Properties & Passenger & Crew & $P$-value \\
\hline Weighted degree & $13.9(5.6-23.7)$ & $8.3(4.4-13.5)$ & $<2.2 \times 10^{-16}$ \\
\hline Eigenvector centrality & $0.3(0.1-0.5)$ & $0.08(0.03-0.2)$ & $<2.2 \times 10^{-16}$ \\
\hline Clustering coefficient & $0.4(0.3-0.4)$ & $0.3(0.2-0.4)$ & $<2.2 \times 10^{-16}$ \\
\hline
\end{tabular}

\section{Analysing the contacts formed during activities}

The total number of contacts made by passengers with passengers from other travelling groups varied according to the type of location and the time spent at that location. The total close contacts plateaued at approximately 3 (IQR 2-5) after spending at least 1 hour in a food and beverage (F\&B) location (Fig. 2A) while the total close contacts was 2 (IQR 1-3) after spending 30 minutes to 1 hour in a sports location and increased to 4 (IQR 2-7) after spending at least 2 hours (Fig. 2C). 




3
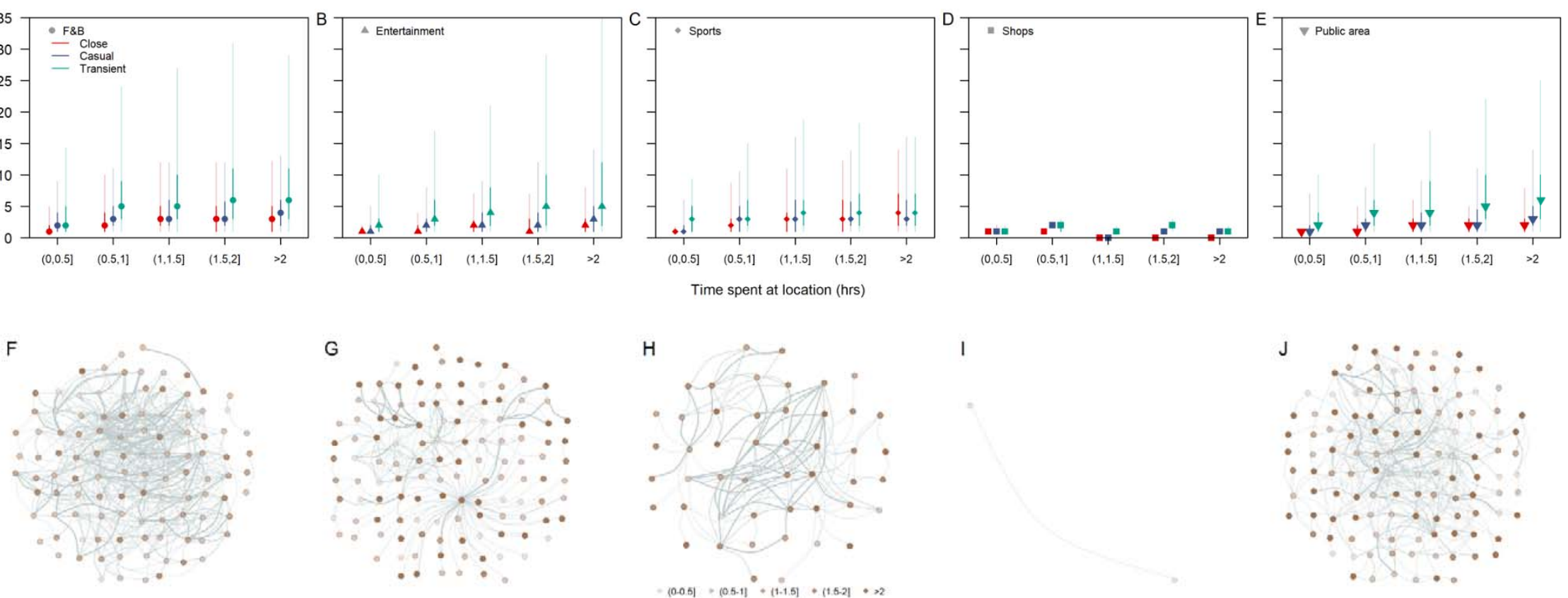

$0.051+105.11 \cdot 0(1.15) \cdot(15.2) \cdot .2$

Fig. 2 Number of contacts made between passengers from different travelling groups per visit to a type of location (A-E) and snapshot of contact network at respective locations for 2 hour intervals on the second day of the sailing (F-J). Type of locations are: F\&B (A, F), entertainment (B, G), sports (C, H), shops (D, I) and public areas (E, J). Median (shapes), $50 \%$ (dark lines) and $95 \%$ intervals (light lines) are shown in (A-E). Nodes of different colour intensity represent the time spent in the location by respective passengers. 
Over the three-day sailings, a median of 71\% (IQR 64-74\%) of all the close contact episodes occurring between passengers from different travelling groups occurred in F\&B locations of which 23\% (IQR 19-26\%) and 38\% (IQR 31-40\%) occurred in the buffet and inclusive restaurants respectively (Fig. 3A and B). 16\% (IQR 11-24\%) of the close contacts occurred in entertainment areas and $8 \%$ (IQR 6-10\%) in sports areas (Fig. 3A). Passengers are largely mask-off when dining or engaged in sports and this accounted for $79 \%$ (IQR $69-84 \%$ ) of all close contact episodes, 69\% (IQR 57-76\%) causal and 60\% (IQR 51-66\%) transient contact episodes (Fig. 3A).
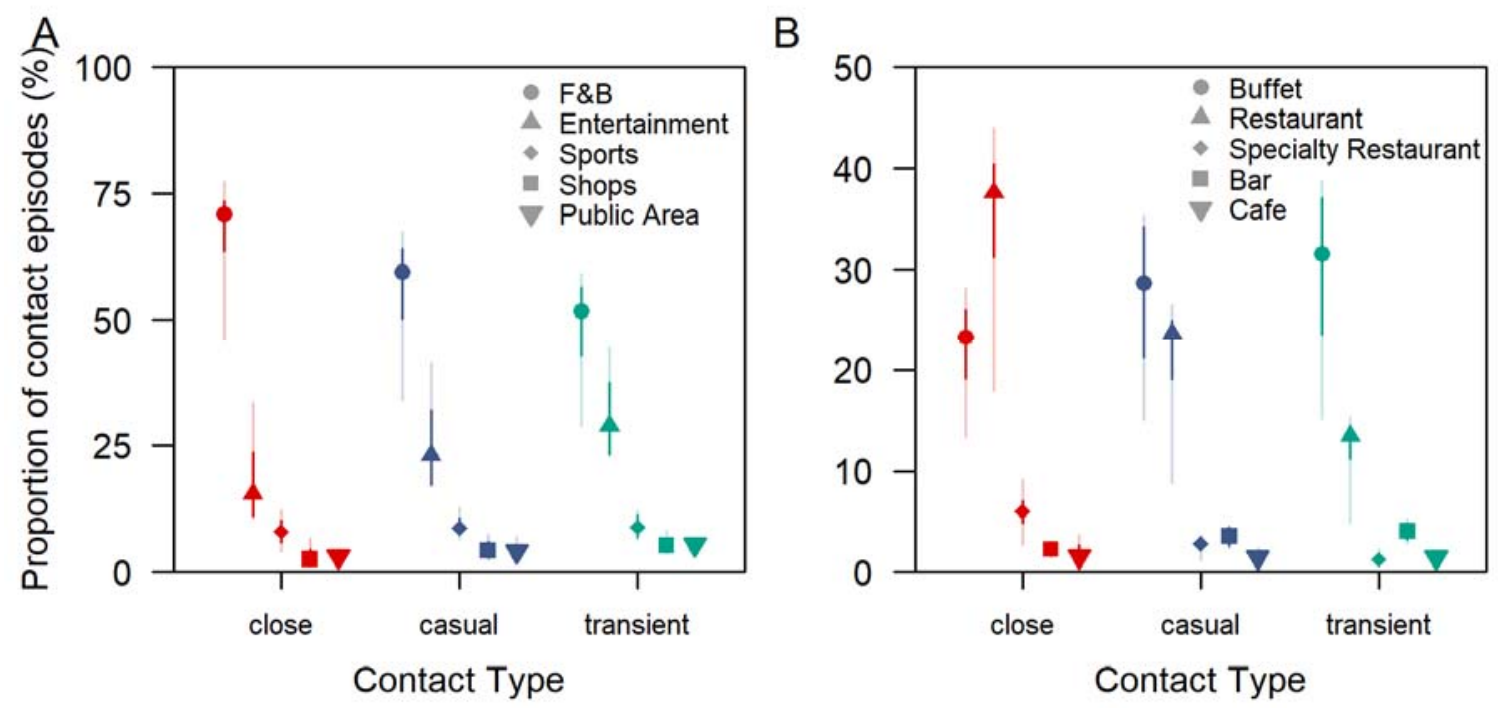

Fig. 3 Type of contact by location of interaction and throughout the sailing, (A) proportion of all close, casual and transient contact episodes between passengers of different travelling groups by the location of interaction, (B) proportion of all close, casual and transient contact episodes between passengers of different travelling groups by respective F\&B locations.

\section{Modelling outbreak dynamics and interventions}

To examine the spread of SARS-CoV-2 on cruise ships and implications for other large-scale multi-day events, we used the contact data to generate an undirected network with nodes and edges representing individuals and the contact between them respectively. We defined the strength of an edge as a function of the proportion of days with recorded contact over a threeday sail period and the mean daily cumulative contact duration between two individuals to approximate a scenario where the edge weight reached $95 \%$ saturation after 3 hours of contact (see Materials and methods). This meant that the propensity for transmission increased and stabilised after 3 hours of contact, to mimic contacts formed in family gatherings over extended periods of time $(14,15)$.

We extended a community network transmission model $(16,17)$ to simulate SAR-CoV-2 Delta variant transmission over seven days, to enable comparison between different interventions during early generations of transmission. We considered interventions including: (i) one-off PCR testing one day before the sailing (to allow for test turnaround time), (ii) rapid antigen testing at 31 the start and halfway through the event, (iii) mask wearing in feasible settings and (iv) 
vaccination coverage among attendees. In both (i) and (ii) testing interventions, we assumed infected individuals were isolated immediately after a positive test. The sensitivity of the PCR and rapid antigen tests were assumed to vary with viral load modelled according to the Delta variant $(8,18-20)$. For the mask wearing intervention, we assumed that passengers of different travelling groups would be exposed to each other without a mask during dining, sports activities (e.g. pool and waterslides, rock climbing, basketball, football) or smoking breaks; and would be wearing a mask correctly otherwise. Furthermore, contacts between passengers and crew were assumed to occur with mask-on at all times and crew-crew contacts were assumed to occur without a mask during meals times, workouts or smoking breaks. The proportion of contacts that occurred without a mask were modelled based on the proportion of contacts occurring in F\&B and sport settings (Fig. 3A).

Under the baseline scenario with no modelled interventions, with one initial infected individual and assuming that the event lasted for 7 days, we estimated a median of 10 individuals (IQR 323) would be infected by the end of the event (Fig. 4A and fig. S3A). Of these, 90\% (IQR 84100 ) would only develop symptoms after the event. Because presymptomatic transmission was assumed to account for $25 \%$ the transmission, more than two generations of infections could sometimes occur during the event (Fig. S3B). We estimated that $64 \%$ and $17 \%$ of the simulated outbreaks involved spillover from passengers to crew and inter-department crew transmission respectively, and we estimated that spillover events first occurred in the 2nd (IQR 2-3) and 3rd (IQR 3-4) generation respectively. Outbreaks with a final size of more than 10 cases occurred in $48 \%$ of our simulations (Fig. $4 \mathrm{~B}$ ).

With the introduction of a one-off PCR test one day prior to the start of the cruise, the index case was isolated in $49 \%$ of the time, while $5 \%$ of the remaining simulations resulted in no transmission due to the stochastic nature of early disease transmission and the structure of the social network (Fig. 4B). As a result, more than half of the simulations had zero secondary cases. The risk of an outbreak of more than 10 cases was reduced to $22 \%$ with the PCR intervention. However, with rapid antigen testing at the start and at halfway through the event instead, only $3 \%$ of simulations resulted in a large outbreak.

We also modelled passenger-passenger interactions occurring under a mask-off setting approximately $60 \%$ of the time (based on the total transient, casual and close contacts in Fig. $2 \mathrm{~A})$ and assumed that all passenger-crew interactions occurred while wearing masks and that $30 \%$ of crew-crew interactions occurred when eating or working out without mask. Under these conditions and in the absence of other interventions, $22 \%$ of the simulations end with a large outbreak size (Fig. 4B). Assuming all individuals onboard the cruise ship were vaccinated (individuals under 12 years of age account for only $2 \%$ of the cruise population), $95 \%$ of simulations resulted in 5 or fewer cases (Fig. 4B).

We examined the expected outbreak size under a combination of interventions under the assumption that vaccination confers $50 \%$ protection against infection $(9,21,22)$ and $50 \%$ lowered infectiousness in a vaccinated but infected individual (23) (Fig. 4C and 4D). Regardless of the testing strategies applied (i.e. no test, once-off PCR test, rapid antigen testing at the start and halfway through the event), and at any level of vaccine coverage, the addition of a mask-on intervention would further reduce the expected outbreak size by about 54\% (IQR 50-59\%). Given outbreak size is the cumulative result of individual transmission events, this implies that the overall intervention effectiveness of a mask mandate is substantially less than the assumed mask-on efficacy at the individual level (Table 4) (24-26). The expected outbreak size in simulations involving rapid antigen testing was less than 1 when vaccine coverage was minimally $25 \%$ (i.e. the expected number of transmission events was less than the initial number 
medRxiv preprint doi: https://doi.org/10.1101/2021.11.12.21266183; this version posted November 14, 2021. The copyright holder for this preprint (which was not certified by peer review) is the author/funder, who has granted medRxiv a license to display the preprint in perpetuity.

It is made available under a CC-BY 4.0 International license .

1 of infected individuals) (Fig. 4C). The expected outbreak size in mask-on, no testing 2 interventions differed from mask-off, once-off PCR testing intervention by less than 1 case 3 across varying vaccination coverage. The same was observed between a mask-on, once off 4 PCR testing intervention and a mask-off, rapid antigen testing intervention. Compared to the 5 expected outbreak size, the $95^{\text {th }}$ percentile of the outbreak size is approximately 3 times higher, 6 with the no testing, mask-off and one-off PCR testing, mask-off interventions generating the 7 highest number of cases among all other combinations of interventions (Fig. 4D).

Sensitivity analysis under different assumptions of the edge weights - and hence per-contact risk - showed an increase in the expected outbreak size as the duration required to be defined as a 'maximal contact' (i.e. weight of one) decreases (Fig. S4). Across all scenarios of varying testing strategy, vaccination coverage, network assumptions for edge weight, the average

13 reduction in the expected outbreak size between a mask-on and mask-off scenario was $60 \%$ 14 (IQR 54-71\%).. Assuming edge weights vary based on the proportion of days over the entire 15 sailing when interactions were recorded (i.e. a transient contact in a day is as risky as a close 16 contact in a day), the difference in the expected outbreak size between a mask-on, no testing 17 scenario and a mask-off, once-off PCR testing widens to 32 cases (IQR 11-64) (Fig. S4C). The 18 difference in the expected outbreak size between a mask-on, once-off PCR testing scenario and 19 a mask-off, rapid antigen testing at the start and halfway through the event differed by 6 cases 20 (IQR 5-19) (Fig. S4C). We obtained similar conclusions on the relative effect of different 21 combinations of interventions when we varied assumptions about the extent of vaccine 22 effectiveness and presymptomatic transmission (see Supplementary Materials). 
medRxiv preprint doi: https://doi.org/10.1101/2021.11.12.21266183; this version posted November 14, 2021. The copyright holder for this preprint (which was not certified by peer review) is the author/funder, who has granted medRxiv a license to display the preprint in perpetuity.

It is made available under a CC-BY 4.0 International license .

A

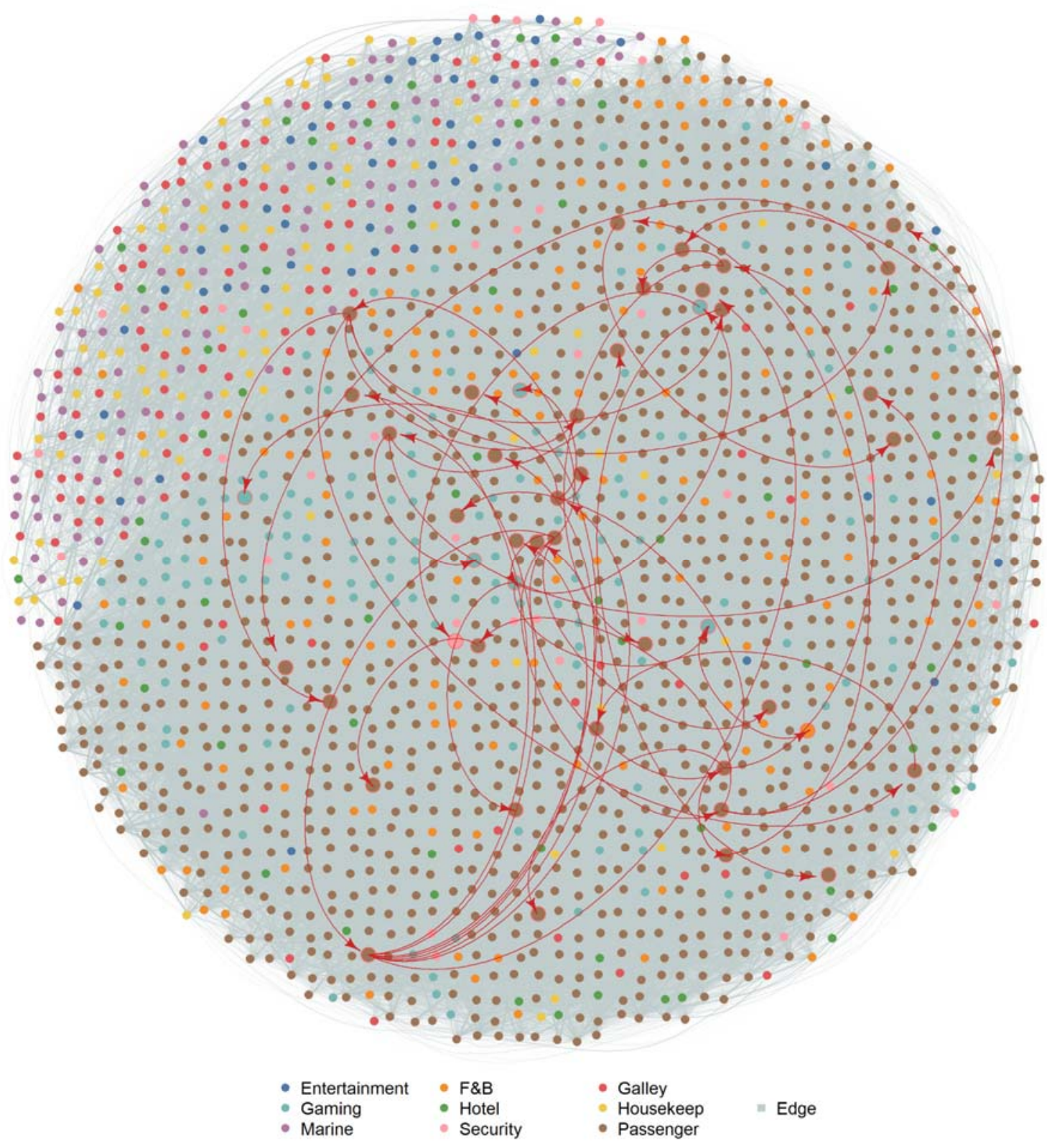

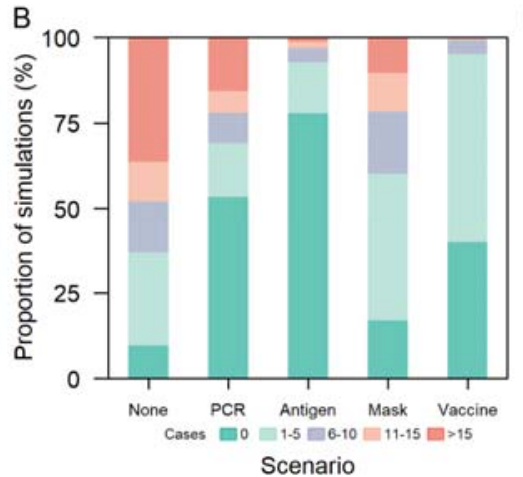

Scenario
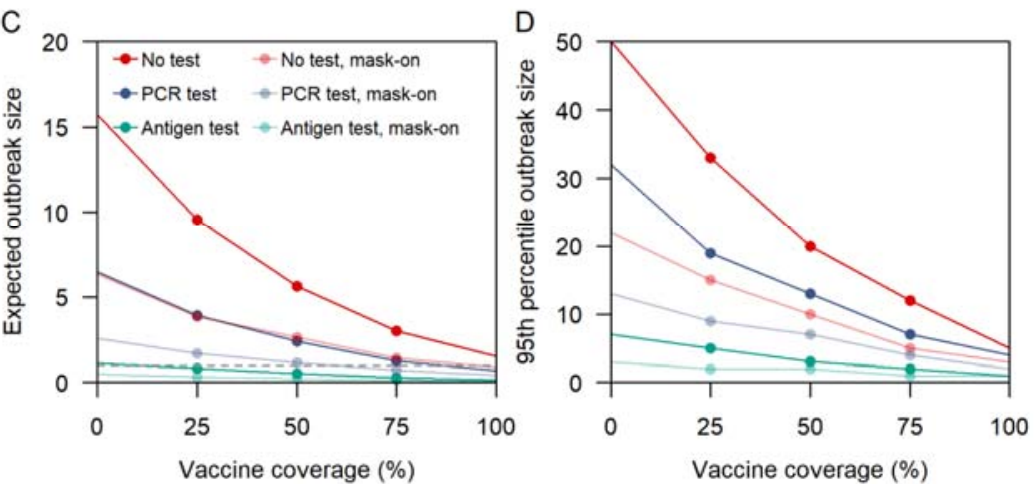
Fig. 4 Outbreak size under respective interventions. (A) Cases and contacts in one outbreak simulation with cases represented by an enlarged node and red curved arrows depicting disease transmission, (B) proportion of simulations by respective outbreak size under different interventions, (C) average outbreak size and (D) 95th percentile of outbreak size for different interventions and varying vaccination coverage.

\section{Discussion}

We found that the structure and intensity of contacts over a multi-day cruise has major consequences for outbreak control in different settings, particularly if there are mask-free activities and leaky testing protocols mean infectious individuals are likely to go undetected. Cruises represent an aggregation of different activities including F\&B, entertainment, sports, meeting, conference, entertainment and workplace settings. The presence of multi-group passengers and crew from different departments can therefore offers insights into the potential dynamics of different actors in other large-scale multi-day events (e.g. a conference where there are participants, organising teams, external vendors, front-end and back-end F\&B service staff, audio visual support teams) and resulting implications for control of SARS-CoV-2.

Our social network analysis showed that passengers had a high number of contacts and their contacts typically exhibit high levels of contact with other individuals. As such, any disease transmission would likely be driven by passenger level interactions rather than crew. In early 2020 , this was evident in the sharp rise in the number of COVID-19 passengers with symptom onset before or during the early stages of quarantine onboard the Diamond Princess (27). While the number of contacts made with other passengers are potentially lowered due to physical distancing and awareness of the pandemic in the studied Singapore setting, the number and type of activities onboard the cruise still means that each passenger forms around 20 unique close contacts per day. Compared to an average of 59 unique close contacts with more than 15 minutes of interaction in a UK community setting over a 14-day period (28), this was 5 times higher, further illustrating the intensity of contacts during such events. More than $70 \%$ of the close and casual contacts on the cruises occurred in F\&B locations where passengers were largely mask-off and thus posing a higher risk of infection and transmission. We observed that the number of close contacts plateaued in F\&B settings as the time spent in the location increases. As such, reducing this risk potentially requires more creative use of space to increase the distance between groups of passengers, improve indoor ventilation and encourage more outdoor dining.

With numerous work functions interfacing with passengers, and given the overlapping shifts and closely related job scope between crew (e.g. F\&B and galley, hotel services and housekeeping), we found it only took around 3 generations for the infection to spread from a passenger to a crew before reaching another crew in a different department. Crew and event personnel play an important role in ensuring smooth operations and their wellbeing should be accounted for in the plans when reopening events. Hence, besides encouraging crew cohorting, interventions that minimise transmission in passengers would have an indirect effect of protecting the crew.

When applied individually, none of the interventions analysed were capable of reducing the expected outbreak size to be lower than one; the number of initial infected cases in passengers, equivalent to a community incidence of 1 per 1,000 individuals. However, a combination of rapid antigen testing at the start and halfway through the sailing with at least $25 \%$ coverage of a vaccine that confers $50 \%$ protection against infection and $50 \%$ lowered infectiousness would result in the cruise event having fewer onward transmission than the number of initial infectives. This is conditioned on the cases exhibiting Delta variant-like high viral loads with prolonged 


\begin{abstract}
shedding $(8,19,20,29)$ which improves the sensitivity of rapid antigen tests. While PCR tests have a higher sensitivity than rapid antigen tests at low viral load levels, the tests need to be conducted on land prior to the event due to the turnaround time required and for validity of lab results. This implies that cases who develop symptoms several days after the sailing may not be identified prior to the event, due to viral loads near the limits of detection, and large outbreaks could occur.
\end{abstract}

The expected outbreak size under different combinations of interventions was sensitive to the assumptions of the network edge weights. When edges are weighted by the proportion of days with recorded interaction over a three-day sail period, two individuals with transient contact in a day are assumed to have the same risk as two individuals with close contact in a day. This assumption is applicable when the dominant mode of transmission is largely independent of the duration of contact (e.g. environmental or airborne transmission). There were 5 times more transient interactions than close contacts and these contacts are now equally at risk of infection. Thus, mask wearing would largely help to lower the risk of transmission and acquiring infection, and outperforms PCR testing or even twice antigen testing interventions especially when the proportion of presymptomatic transmission is high during the early stage of viral shedding.

Both mask-off, rapid antigen testing and mask-on, once-off PCR testing would help to reduce the risk of disease introduction and further transmission if the index cases successfully escape initial detection. While their differences in the expected outbreak size was less than 10 cases across different assumptions to the edge weights, they bring different outcomes to the passenger experience and operations planning - an extra swab test at the middle of the event versus wearing a mask at all times other than during dining and engaged in sports, logistics to check the test outcomes versus monitoring mask wearing practices, managing false positives versus passengers flouting rules.

One limitation to our study was that we did not model contact tracing around detected cases. While cruise lines are trained to trace and quarantine close contacts as part of the pilot reopening, as the ease of rapid testing increases with fast turnaround time, this could serve as a replacement for slower and resource intensive contact tracing in such settings. With presymptomatic transmission of SARS-CoV-2 and high levels of transmissibility of the Delta variant, the effectiveness of contact tracing is approaching a point of saturation in many countries (30). Furthermore, even if the threshold for close contact to be traced is lowered, the corresponding exponential increase in contacts fulfilling this criteria would make it logistically challenging to trace all individuals in a reasonable amount of time. Fully asymptomatic infections - as opposed to presymptomatic infections - were also not considered in the analysis. Should these infections exhibit lowered viral load, the testing interventions would be less likely to detect asymptomatic individuals but any potential for increase in outbreak size would be counteracted by their lowered infectiousness. Currently, there is no strong evidence to suggest that asymptomatic SARS-CoV-2 Delta infections are less infectious than symptomatic individuals $(31,32)$. Lastly, the accuracy of the data collected is largely dependent on the usage behaviour. Passengers are required to carry the contact tracing devices at all times except when engaged in water sports. Hence, interactions at the water sports areas may not be well represented but this effect to our analysis is expected to be minor as the cruise line of study required passengers to book these facilities in advance to facilitate crowd control. In a cabin, each passenger's device may not necessarily be placed in a $2 \mathrm{~m}$ proximity and the frequent close contact interactions in these settings would not be recorded accurately. However, given that individuals in the cabin would largely continue to interact with each other outside the cabin while carrying the device, this would help to record a large proportion of their close interaction. Thus, this limitation is reduced when the probability of infection saturates after a certain level of 
exposure as is in the case of SARS-CoV-2 (33). Despite such limitations, this is one of the few studies with large- and fine-scale data collection from multiple events in one setting and comparison with future studies of similar data collection methods in these settings may help to strengthen our findings.

With the ongoing spread of the highly transmissible SARS-CoV-2 Delta variant alongside increasing vaccination coverage, many countries have oscillated between reopening and restrictions of varying extents, in turn affecting the sustainability of economic and social activities. As the pressure to resume large-scale events increases, but the effectiveness of vaccines against infection and transmission remains variable, combining social interaction data with models such as the one presented here can enable an improved data-driven assessment of the risk of transmission arising from planned activities and the potential reduction offered by the continuation and implementation of non-pharmaceutical interventions.

\section{Materials and methods}

\section{Data}

Each cruise sailing lasts for three days - departing at $7 \mathrm{pm}$ on the first day and arriving at 8am on the last day, and only contacts during this period were studied. Embarking and disembarking begins and ends at approximately 12 noon on both days and devices are stored together prior to issuance or after collection. As such, data prior to departure and after arrival were not used, as the recorded data may be an artefact of devices being stored together.

All individuals onboard a cruise are issued a digital contact tracing device with a unique device identification number. These devices are calibrated based on signal strength to broadcast Bluetooth signals to other devices within a $2 \mathrm{~m}$ radius every 14.9 seconds followed by a scan of nearby signals lasting for 0.1 seconds. Each scan record captures the timestamp of the signal exchange and the identification number of the interacted device. After every five minute interval, the records of 30 unique devices with the highest signal strength in each device are then stored. The stored records are then uploaded to a server on land. Further data processing is required to determine the duration of contact between two individuals. If there are two or more records with consecutive difference(s) of less than five minutes, the duration of contact is the difference of the last and first timestamp in the series of records.

For each cruise sailing, we collected a de-identified manifest with the device identification number and details of the device holder (passenger or crew; for crew: department of the crew; for passengers: cabin number, keycard number, age, gender). The cruise ship can be demarcated into different areas based on the activities in a location (i.e. type of location: food and beverage (F\&B), entertainment, shops, sports, public areas) and all passengers were required to tap-in using their keycards upon entering a new area onboard the cruise ship. We also collected a de-identified list of entry records with each record capturing the keycard number, location and timestamp of entry.

Using the three data sources (i.e. contact data, de-identified manifest and de-identified location records), we categorised the contacts between each dyad into one of four contact groups, namely (i) passenger-passenger contact from within the same travelling group (i.e. passengers in the same cabin or having a cumulative contact duration of more than 5 hours over three days), (ii) passenger-passenger contact from different travelling groups, (iii) crew-crew contact, 
and (vi) passenger-crew contact. 5 hours was selected as a conservative definition for travelling groups, given that this is considerably longer than an average meal duration and more than $99 \%$ of the cumulative contact duration (i.e. sum of all contact episodes) between passengers from different cabins were less than this duration.

We further classified a contact episode in a location into close, casual and transient types of contact if the cumulative duration of contact was at least 15 minutes, at least 5 minutes but less than 15 minutes, and less than 5 minutes respectively in a $2 \mathrm{~m}$ radius (34-36). For each individual in each type of location, we estimated the number of different types of contacts (i.e. close, casual and transient contact) with passengers from different travelling groups over time spent in the location. Across the sailings, for each type of contact, we estimated the proportion of contacts occurring at a type of location over all types of location.

\section{Social Network Construction}

We performed a preliminary social network analysis and estimated the weighted degree distribution (number of contacts made per individual with each contact weighted by the duration of contact, to be elaborated), the distribution of the clustering coefficient (a measure of the triadic linkage among individuals) and individuals' eigenvector centrality (a measure of direct and indirect centrality within a network) of passengers and crew in respective departments in each sailing. We performed a Welch's t-test to evaluate each network property for passengers against that for crew and $p$-values $<0.05$ were considered statistically significant. While the mean and interquartile range (IQR) of each estimate fluctuate across sailings, the $95 \%$ range of the estimates exhibit substantial overlap (Fig. S1). As such, we selected contact data collected over a single sailing with 1208 passengers and 1032 crew to construct the social network for simulating disease transmission. Supplementary simulations were also performed on other sailings to ensure consistency in the percentage reduction in outbreak size for various outbreak interventions across the sailing (Supplementary fig. 7).

In the main analysis, we generated an undirected network with the strength of an edge weighted as a value between 0 and 1 based on the proportion of days with recorded contact over a threeday sail period and the exponent transformation of the mean daily cumulative contact duration between two individuals as follows:

$$
w_{i j}=c_{i j}\left(1-e^{-\overline{d_{l j}} \sigma}\right)
$$

where $w_{i j}$ is the weight of a contact between individuals $i$ and $j, c_{i j}$ is the proportion of days with recorded contact and $\overline{d_{l}}$ is the mean daily cumulative contact duration expressed hours. $\sigma$ is a scalar of 0.5 to approximate a scenario where the edge weight reaches $95 \%$ saturation after 3 hours of contact $\left(w_{i j} \rightarrow 1\right)$. As a sensitivity analysis, we explored other weightings for the network edges; similar to the above but $95 \%$ saturation to the same level of infection risk after 1 hour of contact, or based on the proportion of days over the entire sailing with recorded contact only. These scenarios depict how risk of infection increases based on contact duration as observed in SARS-CoV-2 outbreaks in settings of poor ventilation $(37,38)$ or transmission driven by a highly transmissible pathogen onboard cruises such as norovirus (39). 
We simulated SARS-CoV-2 Delta variant transmission on the above generated social contact network by extending the individual based models developed by Firth et al and Hellewell et al (Table 3) $(16,17)$.

Table 3 Parameter values and assumptions

\begin{tabular}{|l|l|l|}
\hline Parameter & Assumed values & Details and references \\
\hline Incubation period (days) & $\begin{array}{l}\text { Mean }=4.4, \\
\text { sd =1.9 }\end{array}$ & $(40)$ \\
\hline $\begin{array}{l}\text { Adherence to isolation } \\
\text { when tested positive (\%) }\end{array}$ & 100 & $\begin{array}{l}\text { For scenarios involving testing only and we } \\
\text { assume that there are available cabins for } \\
\text { individuals to isolate given that cruises are } \\
\text { operating at 50\% capacity. }\end{array}$ \\
\hline $\begin{array}{l}\text { Delay from positive test } \\
\text { to isolation (hrs) }\end{array}$ & No delay & $\begin{array}{l}\text { For scenarios involving testing, individuals } \\
\text { were isolated once tested positive. }\end{array}$ \\
\hline $\begin{array}{l}\text { Initial cases among } \\
\text { passengers }\end{array}$ & 1 & $\begin{array}{l}\text { Each network formulation uses one scaling } \\
\text { parameter value to calibrate the probability of } \\
\text { Delta infection among cabin contacts to be } \\
\text { similar to that of household contacts of } 20 \% \\
\text { (29, 41, 42). The range of values used across } \\
\text { the different network formulations are as } \\
\text { shown. }\end{array}$ \\
\hline Scaling parameter & $0.24-0.26$ & \multicolumn{2}{|l}{} \\
\hline
\end{tabular}

For each simulation, we assume that the disease is introduced by one passenger who could be infected up to 14 days prior to the event, with equal probability on any of the days but the onset of the index case would only occur between the start (i.e. day 1) and the end (i.e. day 7) of the event. The distribution of the symptoms onset date, $S$, on respective day of the event, $d$, is as follows:

$$
S(d)=\int_{-13}^{0} I(\delta) \theta(d+|\delta|) d \delta
$$

where $\delta$ is the day of infection prior to the event (i.e. $\delta=0$ represents the day before the start of event), $I(\delta)$ is the probability of being infected on any of the 14 days prior to the event and is fixed at $1 / 14, \theta$ is the incubation period distribution with $d+|\delta|$ representing the time since infection on the respective day of the event.

Currently, all crew are required to be tested weekly and are largely confined to the cruise except during periods of shore leave, thereby reducing the risk of disease introduction by crew. Each day, the model searches for susceptible individuals in contact with the infected cases who are 
not isolated and infection from infector $i$ to susceptible individual $j$ occurs based on the following probability:

$$
P_{i \rightarrow j}(d)=1-e^{-\Delta d \lambda_{i \rightarrow j}(d)}
$$

where $\Delta d$ is the modelled time step of one day, and $\lambda_{i \rightarrow j}(d)$ is the force of infection between infector $i$ and susceptible individual $j$ on day $d$ expressed as:

$$
\lambda_{i \rightarrow j}(d)=w_{i j} f\left(d \mid \mu_{i}, \alpha_{i}, \omega_{i}\right) r_{\text {scale }} \beta_{i j}
$$

where $f\left(d \mid \mu_{i}, \alpha_{i}, \omega_{i}\right)$ is the probability density function that represents the infectiousness of the infector on day $d$. We assumed a skew normal distribution with location parameter $\mu_{i}$ set based on the infector's day of onset, a slant parameter $\alpha_{i}$ and a scale parameter $\omega_{i}$ adjusted such that $25 \%$ of the infections occurred prior to symptom onset. As there is substantial uncertainty in the proportion of presymptomatic transmission for SARS-CoV-2 (43), for sensitivity analysis, we considered a scenario where about $50 \%$ of transmission occurred prior to symptom onset.

We multiplied the force of infection with a scaling factor, $r_{\text {scale }}$, so that the mean probability of infection of a susceptible individual staying in the same cabin as an infected case is approximately $20 \%$ assuming exposure in the cabin and during all shared activities throughout the entire duration of infectiousness, similar to the household attack rates for SARS-CoV-2 Delta variant cases $(29,41) . \beta_{i j}$ is the relative risk of infection depending on vaccination status and mask wearing behaviours, and is parameterised to reduce the probability of infection according to Table 4.

Table 4 Parameter values for the relative risk of infection, $\beta_{i j}$

\begin{tabular}{|c|l|l|}
\hline Relative risk & Vaccination and mask wearing status & Remarks \\
\hline$\beta=1$ & $\begin{array}{l}\text { Mask-off (i.e not wearing a mask) and } \\
\text { both the infected and susceptible } \\
\text { individuals are not vaccinated. }\end{array}$ & $\begin{array}{l}\text { No change in probability of } \\
\text { infection. }\end{array}$ \\
\hline$\beta_{v i}$ & $\begin{array}{l}\text { Mask-off and infected individual } i \text { is } \\
\text { vaccinated. }\end{array}$ & $\begin{array}{l}\text { Mean probability of } \\
\text { transmitting infection reduces } \\
\text { by 50\% (23). }\end{array}$ \\
\hline$\beta_{v j}$ & $\begin{array}{l}\text { Mask-off and susceptible individual } j \\
\text { is vaccinated. }\end{array}$ & $\begin{array}{l}\text { Mean probability of acquiring } \\
\text { infection reduces by 50\% (9, } \\
21,22) .\end{array}$ \\
\hline$\beta_{v i j}$ & $\begin{array}{l}\text { Mask-off and both infected individual } \\
i \text { and susceptible individual } j \text { are } \\
\text { vaccinated. }\end{array}$ & $\begin{array}{l}\text { Mean probability of infection } \\
\text { reduces by 75\%. Assumes } \\
\text { the effect of vaccination on } \\
\text { transmission and acquiring } \\
\text { infection is independent. }\end{array}$ \\
\hline$\beta_{m}$ & Mask-on (i.e. wearing a mask) only. & $\begin{array}{l}\text { Mean probability of infection } \\
\text { reduces by 80\% when both } \\
\text { the infected individual and }\end{array}$ \\
\hline
\end{tabular}




\begin{tabular}{|c|l|l|}
\hline & & $\begin{array}{l}\text { susceptible contact are } \\
\text { wearing a mask (24). }\end{array}$ \\
\hline$\beta_{m, v i}$ & $\begin{array}{l}\text { Mask-on and infected individual } i \text { is } \\
\text { vaccinated. }\end{array}$ & $\begin{array}{l}\text { Mean probability of infection } \\
\text { reduces by 90\%. Assumes } \\
\text { the effect of vaccination and } \\
\text { mask wearing on reducing } \\
\text { the probability of transmitting } \\
\text { infection is independent. }\end{array}$ \\
\hline$\beta_{m, v j}$ & $\begin{array}{l}\text { Mask-on and susceptible individual } \\
j \text { is vaccinated. }\end{array}$ & $\begin{array}{l}\text { Mean probability of infection } \\
\text { reduces by 90\%. Assumes } \\
\text { the effect of vaccination and } \\
\text { mask wearing on reducing } \\
\text { the probability of acquiring } \\
\text { infection is independent. }\end{array}$ \\
\hline$\beta_{m, v i j}$ & $\begin{array}{l}\text { Mask-on and both infected individual } \\
i \text { and susceptible individual } j \text { are } \\
\text { vaccinated. }\end{array}$ & $\begin{array}{l}\text { Mean probability of infection } \\
\text { reduces by 95\%. Assumes } \\
\text { the effect of vaccination and } \\
\text { mask wearing on reducing } \\
\text { the probability of transmission } \\
\text { and acquiring infection is } \\
\text { independent. }\end{array}$ \\
\hline
\end{tabular}

\section{Interventions}

3 In the testing interventions, the sensitivity of the tests were assumed to vary with viral load. We

4 assumed PCR is $100 \%$ sensitive for cycle threshold (Ct) values (a measure of viral load) below 535 and rapid antigen tests is $94.5 \%$ sensitive for $\mathrm{Ct}$ values below 25 and lowered sensitivities as 6 the $\mathrm{Ct}$ values increases (18). The viral load trajectory was modelled in relation to the Delta 7 variant, rising above the limits of test detection three days before symptoms onset with 8 prolonged shedding post symptoms onset $(8,19,20)$.

For the mask wearing intervention, the expected weight of the contact between individuals $i$ and vaccination as follows:

where $m_{g}$ is the probability that the contact between any pairs of individual of a contact group

$$
\begin{gathered}
\bar{\lambda}_{i j g}(t)=w_{i j} \int_{t-1}^{t} f\left(u ; \mu_{i}, \alpha_{i}, \omega_{i}\right) d u r_{s c a l e}\left[\left(1-m_{g}\right) \beta_{v}+m_{g} \beta_{m, v}\right] \\
\beta_{v} \in\left\{\beta, \beta_{v i}, \beta_{v j}, \beta_{v i j}\right\} \\
\beta_{m, v} \in\left\{\beta_{m}, \beta_{m, v i}, \beta_{m, v j}, \beta_{m, v i j}\right\}
\end{gathered}
$$
goccurs while wearing a mask. $\beta_{v}$ and $\beta_{m, v}$ are the relative risk of infection based on the vaccination status of the infector and infectee (Table 2). 
medRxiv preprint doi: https://doi.org/10.1101/2021.11.12.21266183; this version posted November 14, 2021. The copyright holder for this preprint (which was not certified by peer review) is the author/funder, who has granted medRxiv a license to display the preprint in perpetuity. It is made available under a CC-BY 4.0 International license .

1

2 For each intervention or combination of interventions, we ran 1000 simulations. We estimated

3 the incidence by the day of infection, the number of cases in each generation, and the expected

4 final outbreak size. All analyses were done in R version 4.0 .4 (44) and the model code is 5 available at (45).

6 


\section{References}

1. A. Pavli, H. C. Maltezou, COVID-19 vaccine passport for safe resumption of travel. Journal of Travel Medicine. 28 (2021), doi:10.1093/jtm/taab079.

2. D. for D. GOV.UK Culture, Media and Sport, Events Research Programme: Phase I findings. GOV.UK (2021), (available at https://www.gov.uk/government/publications/events-research-programme-phase-ifindings/events-research-programme-phase-i-findings).

3. The Lancet Microbe, Vaccine certificates: does the end justify the means? The Lancet Microbe. 2, e130 (2021).

4. Singapore Tourism Board, Third consecutive year of growth for Singapore tourism sector in 2018 | STB (2019), (available at https://www.stb.gov.sg/content/stb/en/mediacentre/media-releases/third-consecutive-year-of-growth-for-singapore-tourism-sectorin-2018.html).

5. A. Curley, R. Garber, V. Krishnan, J. Tellez, For corporate travel, a long recovery ahead, (available at http://ceros.mckinsey.com/coronavirus-promo-video-desktop).

6. Environment Modelling Group, Department for Digital, Culture, Media and Sport, EMG and DCMS: Science framework for opening up group events, 16 March 2021. GOV.UK (2021), (available at https://www.gov.uk/government/publications/emg-and-dcmsscience-framework-for-opening-up-group-events-16-march-2021).

7. B. Li, A. Deng, K. Li, Y. Hu, Z. Li, Q. Xiong, Z. Liu, Q. Guo, L. Zou, H. Zhang, M. Zhang, F. Ouyang, J. Su, W. Su, J. Xu, H. Lin, J. Sun, J. Peng, H. Jiang, P. Zhou, T. Hu, M. Luo, Y. Zhang, H. Zheng, J. Xiao, T. Liu, R. Che, H. Zeng, Z. Zheng, Y. Huang, J. Yu, L. Yi, J. Wu, J. Chen, H. Zhong, X. Deng, M. Kang, O. G. Pybus, M. Hall, K. A. Lythgoe, Y. Li, J. Yuan, J. He, J. Lu, Virological, in press, doi:10.1101/2021.07.07.21260122.

8. S. W. X. Ong, C. J. Chiew, L. W. Ang, T.-M. Mak, L. Cui, M. P. H. S. Toh, Y. D. Lim, P. H. Lee, T. H. Lee, P. Y. Chia, S. Maurer-Stroh, R. T. P. Lin, Y.-S. Leo, V. J. Lee, D. C. Lye, B. E. Young, Clinical and virological features of SARS-CoV-2 variants of concern: a retrospective cohort study comparing B.1.1.7 (Alpha), B.1.315 (Beta), and B.1.617.2 (Delta). Clinical Infectious Diseases (2021), doi:10.1093/cid/ciab721.

9. P. Elliott, D. Haw, H. Wang, O. Eales, C. Walters, K. Ainslie, C. Atchison, C. Fronterre, P. Diggle, A. Page, A. Trotter, S. Prosolek, T. (cog-Uk), D. Ashby, C. Donnelly, W. Barclay, G. Cooke, H. Ward, A. Darzi, S. Riley, "REACT-1 round 13 final report: exponential growth, high prevalence of SARS-CoV-2 and vaccine effectiveness associated with Delta variant in England during May to July 2021" (Working Paper, 2021), (available at http://spiral.imperial.ac.uk/handle/10044/1/90800).

10. L. Ferretti, C. Wymant, M. Kendall, L. Zhao, A. Nurtay, L. Abeler-Dörner, M. Parker, D. Bonsall, C. Fraser, Quantifying SARS-CoV-2 transmission suggests epidemic control with digital contact tracing. Science. 368, eabb6936 (2020).

11. J. O'Connell, D. T. O'Keeffe, Contact Tracing for Covid-19 - A Digital Inoculation against Future Pandemics. New England Journal of Medicine. 385, 484-487 (2021).

12. J. A. E. Smith, S. Hopkins, C. Turner, K. Dack, A. Trelfa, P. S. Monks, Public Health Impact of Mass Sporting and Cultural Events in a rising COVID-19 prevalence in England, 15 (2021).

13. K. Eames, S. Bansal, S. Frost, S. Riley, Six challenges in measuring contact networks for use in modelling. Epidemics. 10, 72-77 (2015).

14. S. E. F. Yong, D. E. Anderson, W. E. Wei, J. Pang, W. N. Chia, C. W. Tan, Y. L. Teoh, P. Rajendram, M. P. H. S. Toh, C. Poh, V. T. J. Koh, J. Lum, N.-A. M. Suhaimi, P. Y. Chia, M. I.-C. Chen, S. Vasoo, B. Ong, Y. S. Leo, L. Wang, V. J. M. Lee, Connecting clusters of COVID-19: an epidemiological and serological investigation. The Lancet Infectious Diseases. 20, 809-815 (2020).

15. Y. Liu, R. M. Eggo, A. J. Kucharski, Secondary attack rate and superspreading events 
for SARS-CoV-2. The Lancet. 395, e47 (2020).

16. J. A. Firth, J. Hellewell, P. Klepac, S. Kissler, A. J. Kucharski, L. G. Spurgin, Using a real-world network to model localized COVID-19 control strategies. Nat Med. 26, 16161622 (2020).

17. J. Hellewell, S. Abbott, A. Gimma, N. I. Bosse, C. I. Jarvis, T. W. Russell, J. D. Munday, A. J. Kucharski, W. J. Edmunds, F. Sun, S. Flasche, B. J. Quilty, N. Davies, Y. Liu, S. Clifford, P. Klepac, M. Jit, C. Diamond, H. Gibbs, K. van Zandvoort, S. Funk, R. M. Eggo, Feasibility of controlling COVID-19 outbreaks by isolation of cases and contacts. The Lancet Global Health. 8, e488-e496 (2020).

18. J. Dinnes, J. J. Deeks, S. Berhane, M. Taylor, A. Adriano, C. Davenport, S. Dittrich, D. Emperador, Y. Takwoingi, J. Cunningham, S. Beese, J. Domen, J. Dretzke, L. F. di Ruffano, I. M. Harris, M. J. Price, S. Taylor-Phillips, L. Hooft, M. M. Leeflang, M. D. Mclnnes, R. Spijker, A. V. den Bruel, C. C.-19 D. T. A. Group, Rapid, point-of-care antigen and molecular-based tests for diagnosis of SARS-CoV-2 infection. Cochrane Database of Systematic Reviews (2021), doi:10.1002/14651858.CD013705.pub2.

19. P. Y. Chia, S. W. X. Ong, C. J. Chiew, L. W. Ang, J.-M. Chavatte, T.-M. Mak, L. Cui, S. Kalimuddin, W. N. Chia, C. W. Tan, L. Y. A. Chai, S. Y. Tan, S. Zheng, R. T. P. Lin, L. Wang, Y.-S. Leo, V. J. Lee, D. C. Lye, B. E. Young, "Virological and serological kinetics of SARS-CoV-2 Delta variant vaccine-breakthrough infections: a multi-center cohort study" (2021), p. 2021.07.28.21261295, , doi:10.1101/2021.07.28.21261295.

20. S. M. Kissler, J. R. Fauver, C. Mack, C. G. Tai, M. I. Breban, A. E. Watkins, R. M. Samant, D. J. Anderson, J. Metti, G. Khullar, R. Baits, M. MacKay, D. Salgado, T. Baker, J. T. Dudley, C. E. Mason, D. D. Ho, N. D. Grubaugh, Y. H. Grad, "Viral dynamics of SARS-CoV-2 variants in vaccinated and unvaccinated individuals" (2021), p. 2021.02.16.21251535, , doi:10.1101/2021.02.16.21251535.

21. S. Nanduri, Effectiveness of Pfizer-BioNTech and Moderna Vaccines in Preventing SARS-CoV-2 Infection Among Nursing Home Residents Before and During Widespread Circulation of the SARS-CoV-2 B.1.617.2 (Delta) Variant - National Healthcare Safety Network, March 1-August 1, 2021. MMWR Morb Mortal Wkly Rep. 70, 1163-1166 (2021).

22. A. Fowlkes, Effectiveness of COVID-19 Vaccines in Preventing SARS-CoV-2 Infection Among Frontline Workers Before and During B.1.617.2 (Delta) Variant Predominance - Eight U.S. Locations, December 2020-August 2021. MMWR Morb Mortal Wkly Rep. 70, 1167-1169 (2021).

23. R. J. Harris, J. A. Hall, A. Zaidi, N. J. Andrews, J. K. Dunbar, G. Dabrera, Effect of Vaccination on Household Transmission of SARS-CoV-2 in England. New England Journal of Medicine. 385, 759-760 (2021).

24. D. K. Chu, E. A. Akl, S. Duda, K. Solo, S. Yaacoub, H. J. Schünemann, D. K. Chu, E. A. Akl, A. El-harakeh, A. Bognanni, T. Lotfi, M. Loeb, A. Hajizadeh, A. Bak, A. Izcovich, C. A. Cuello-Garcia, C. Chen, D. J. Harris, E. Borowiack, F. Chamseddine, F. Schünemann, G. P. Morgano, G. E. U. M. Schünemann, G. Chen, H. Zhao, I. Neumann, J. Chan, J. Khabsa, L. Hneiny, L. Harrison, M. Smith, N. Rizk, P. G. Rossi, P. AbiHanna, R. El-khoury, R. Stalteri, T. Baldeh, T. Piggott, Y. Zhang, Z. Saad, A. Khamis, M. Reinap, S. Duda, K. Solo, S. Yaacoub, H. J. Schünemann, Physical distancing, face masks, and eye protection to prevent person-to-person transmission of SARS-CoV-2 and COVID-19: a systematic review and meta-analysis. The Lancet. 395, 1973-1987 (2020).

25. R. Yapp, Z. Willis, J. Jones, Coronavirus (COVID-19) Infection Survey technical article: analysis of populations in the UK by risk of testing positive for COVID-19, September 2021 (2021), (available at

https://www.ons.gov.uk/peoplepopulationandcommunity/healthandsocialcare/conditions anddiseases/articles/coronaviruscovid19infectionsurveytechnicalarticle/analysisofpopul ationsintheukbyriskoftestingpositiveforcovid19september2021).

26. J. Abaluck, L. H. Kwong, A. Styczynski, A. Haque, M. A. Kabir, E. Bates-Jefferys, E. Crawford, J. Benjamin-Chung, S. Benhachmi, S. Raihan, S. Rahman, N. Zaman, L. 
Stephen, M. Mobarak, M. A. Haque, "The Impact of Community Masking on COVID-19: A Cluster-Randomized Trial in Bangladesh" (2021), (available at https://www.povertyaction.org/publication/impact-community-masking-covid-19-cluster-randomized-trialbangladesh).

27. Expert Taskforce for the COVID-19 Cruise Ship Outbreak, Epidemiology of COVID-19 Outbreak on Cruise Ship Quarantined at Yokohama, Japan, February 2020 - Volume 26, Number 11-November 2020 - Emerging Infectious Diseases journal - CDC. Emerg Infect Dis. 26, 2591-2597 (2020).

28. M. J. Keeling, T. D. Hollingsworth, J. M. Read, Efficacy of contact tracing for the containment of the 2019 novel coronavirus (COVID-19). J Epidemiol Community Health. 74, 861-866 (2020).

29. M. Kang, H. Xin, J. Yuan, S. T. Ali, Z. Liang, J. Zhang, T. Hu, E. H. Y. Lau, Y. Zhang, M. Zhang, B. J. Cowling, Y. Li, P. Wu, "Transmission dynamics and epidemiological characteristics of Delta variant infections in China" (2021), p. 2021.08.12.21261991, , doi:10.1101/2021.08.12.21261991.

30. S. Reardon, How the Delta variant achieves its ultrafast spread. Nature (2021), doi:10.1038/d41586-021-01986-w.

31. M. Stockbridge, M. Purver, T. Solel, A. Jian, D. Arikan, R. Ovens, A.

Swayamprakasam, D. Chapman, R. Vipond, A. Bown, A. Sienkiewicz, S. Tunkel, T. A. Fowler, T. E. Peto, S. Hopkins, Technical Report: In vitro and clinical post-market surveillance of Biotime SARS-CoV-2 Lateral Flow Antigen Device in detecting the SARS-CoV-2 Delta variant (B.1.617.2) (2021), (available at https://assets.publishing.service.gov.uk/government/uploads/system/uploads/attachme nt_data/file/999867/in-vitro-and-clinical-post-market-surveillance-of-Biotime-SARSCoV-2-Lateral-Flow-Antigen-Device-in-detecting-the-SARS-CoV-2-Delta-variantB.1.617.2.pdf).

32. Abbott, Evaluating Delta and other COVID variants to ensure test effectiveness. Abbott, (available at https://www.abbott.com/corpnewsroom/diagnostics-testing/monitoringcovid-variants-to-ensure-test-effectiveness.html).

33. X. Zhang, J. Wang, Dose-response Relation Deduced for Coronaviruses From Coronavirus Disease 2019, Severe Acute Respiratory Syndrome, and Middle East Respiratory Syndrome: Meta-analysis Results and its Application for Infection Risk Assessment of Aerosol Transmission. Clinical Infectious Diseases. 73, e241-e245 (2021).

34. Government of the United Kingdom, Guidance for contacts of people with confirmed coronavirus (COVID-19) infection who do not live with the person. GOV.UK (2021), (available at https://www.gov.uk/government/publications/guidance-for-contacts-ofpeople-with-possible-or-confirmed-coronavirus-covid-19-infection-who-do-not-live-withthe-person/guidance-for-contacts-of-people-with-possible-or-confirmed-coronaviruscovid-19-infection-who-do-not-live-with-the-person).

35. Ministry of Health, Singapore, MOH | FAQs - Confirmed Cases and Contact Tracing (2021), (available at https://www.moh.gov.sg/covid-19/general/faqs---confirmed-casesand-contact-tracing).

36. Centres for Disease Control and Prevention, Public Health Guidance for CommunityRelated Exposure. Centers for Disease Control and Prevention (2020), (available at https://www.cdc.gov/coronavirus/2019-ncov/php/public-health-recommendations.html).

37. J. Lu, J. Gu, K. Li, C. Xu, W. Su, Z. Lai, D. Zhou, C. Yu, B. Xu, Z. Yang, COVID-19 Outbreak Associated with Air Conditioning in Restaurant, Guangzhou, China, 2020 Volume 26, Number 7-July 2020 - Emerging Infectious Diseases journal - CDC, doi:10.3201/eid2607.200764.

38. Y. Shen, C. Li, H. Dong, Z. Wang, L. Martinez, Z. Sun, A. Handel, Z. Chen, E. Chen, M. H. Ebell, F. Wang, B. Yi, H. Wang, X. Wang, A. Wang, B. Chen, Y. Qi, L. Liang, Y. Li, F. Ling, J. Chen, G. Xu, Community Outbreak Investigation of SARS-CoV-2 Transmission Among Bus Riders in Eastern China. JAMA Internal Medicine. 180, 1665-1671 (2020). 
39. E. T. Isakbaeva, M.-A. Widdowson, R. S. Beard, S. N. Bulens, J. Mullins, S. S. Monroe, J. Bresee, P. Sassano, E. H. Cramer, R. I. Glass, Norovirus Transmission on Cruise Ship. Emerg Infect Dis. 11, 154-157 (2005).

40. M. Zhang, J. Xiao, A. Deng, Y. Zhang, Y. Zhuang, T. Hu, J. Li, H. Tu, B. Li, Y. Zhou, J. Yuan, L. Luo, Z. Liang, Y. Huang, G. Ye, M. Cai, G. Li, B. Yang, B. Xu, X. Huang, Y. Cui, D. Ren, Y. Zhang, M. Kang, Y. Li, Transmission Dynamics of an Outbreak of the COVID-19 Delta Variant B.1.617.2 - Guangdong Province, China, May-June 2021. CCDCW. 3, 584-586 (2021).

41. Public Health England, "SARS-CoV-2 variants of concern and variants under investigation in England Technical briefing 14" (2021), (available at https://assets.publishing.service.gov.uk/government/uploads/system/uploads/attachme nt_data/file/991343/Variants_of_Concern_VOC_Technical_Briefing_14.pdf).

42. O. T. Ng, V. Koh, C. J. Chiew, K. Marimuthu, N. M. Thevasagayam, T. M. Mak, J. K. Chua, S. S. H. Ong, Y. K. Lim, Z. Ferdous, A. K. bte Johari, M. I.-C. Chen, S. MaurerStroh, C. Lin, R. T. P. Lin, K. B. Tan, A. R. Cook, Y. S. Leo, V. J. Lee (in press), Impact of Delta Variant and Vaccination on SARS-CoV-2 Secondary Attack Rate Among Household Close Contacts. MMWR Morb Mortal Wkly Rep.

43. D. Buitrago-Garcia, D. Egli-Gany, M. J. Counotte, S. Hossmann, H. Imeri, A. M. Ipekci, G. Salanti, N. Low, Occurrence and transmission potential of asymptomatic and presymptomatic SARS-CoV-2 infections: A living systematic review and meta-analysis. PLoS Med. 17, e1003346 (2020).

44. R Core Team, R: A Language and Environment for Statistical Computing ( $\mathrm{R}$ Foundation for Statistical Computing, Vienna, Austria, 2020; https://www.Rproject.org/).

45. R. Pung, Code for Using high-resolution contact networks to evaluate SARS-CoV-2 transmission and control in large-scale multi-day events (2021;

https://github.com/rachaelpung/cruise_networks).

\section{Funding}

RP declares funding from the Singapore Ministry of Health. AJK declares funding from Wellcome Trust $((206250 / Z / 17 / Z)$ and the National Institute of Health Research (NIHR)(NIHR200908).

Author contributions: Conceptualization: RP, VJL, AJK. Methodology: RP, JAF, LGS, AJK. Investigation: RP, Singapore CruiseSafe working group. Visualization: RP, JAF, LGS, AJK. Supervision: VJL, AJK. Writing, original draft: RP, AJK. Writing, review \& editing: all authors

Competing interests: Authors declare that they have no competing interests.

Data and materials availability: All data are available in the manuscript or the supplementary materials. The data and code used for our analyses is publicly available at (45). 